\title{
Severe malignant hypertension following renal artery embolization: a crucial role for the renal microcirculation in the pathogenesis of hypertension?
}

\begin{abstract}
Malignant hypertension is the most severe form of hypertension that is usually fatal if not properly managed. It is usually associated with evidence of microvascular damage such as retinopathy and nephropathy. Renal artery embolization is a widely utilised tool for the management of a wide range of conditions including drug resistant renovascular hypertension in patients with end stage renal failure. In this report we describe two patients with mild-to-moderate hypertension who underwent renal artery embolization for reasons unrelated to their hypertension. Contrary to conventional wisdom, in both patients hypertension became more severe and difficult to control. This report describes the cases and discusses the implications for current theory and the possible role of the microcirculation in the causation of hypertension.
\end{abstract}

Volume 9 Issue $3-2017$

\author{
Nahida Khan,' John Jeans,' Seyed Mahdi, ${ }^{2}$ \\ Anna Maria Belli, ${ }^{2}$ Tarek F Antonios ${ }^{1,2}$ \\ 'Blood Pressure Unit, UK \\ 2Department of Radiology, St. George?s University Hospitals \\ NHS Foundation Trust, UK \\ ${ }^{3}$ Molecular \& Clinical Sciences Research Institute, St. George?s, \\ University of London, UK
}

\begin{abstract}
Correspondence: Tarek FT Antonios, Molecular \& Clinical Sciences Research Institute, St George $\square$ s, University of London, London SWI7 ORE, UK, Tel 2087255627, Fax 2087252722
\end{abstract} Email t.antonios@sgul.ac.uk

Received: June 28, 2017 | Published: July 18, 2017

\section{Introduction}

Renal artery embolization (RAE) is a widely utilised tool for management of a wide range of conditions. Initially described in 1971, it was first employed in embolization of entire kidneys for renal cell carcinoma ${ }^{1}$ and drug resistant renovascular hypertension in patients with end stage renal failure. ${ }^{2}$ Subsequently, partial (selective) embolization was used for arteriovenous malformations, pseudoaneurysms, and large angiomyolipomas at risk of haemorrhage. More recently, targeted embolization is being used for traumatic renal injuries.

Current thinking is that embolization of the kidney induces necrosis of the renal parenchyma. This stops the production of renin in the juxtaglomerular apparatus and therefore prevents the activation of the renin-angiotensin-aldosterone (RAS) system -- a potent cause of hypertension. Published literature reports cases where embolization of renal parenchyma that is distal to a focal stenosis or proximal to non-functioning renal tissue has substantially reduced systemic blood pressure (BP) ${ }^{3,4}$ Recently, two patients with hypertension underwent renal artery embolization for reasons unrelated to their hypertension. Contrary to conventional wisdom, in both patients hypertension became more severe and difficult to control. This article will describe the cases and discuss the implications for current theory and practice.

\section{Case I}

A 39-year-old normotensive female patient of Australian descent was admitted to a London hospital with severe abdominal and loin pain. She underwent an abdominal ultrasound scan, which revealed that suffered a retroperitoneal bleeding secondary to a ruptured angiomyolipoma from her left kidney. The patient was initially managed conservatively with painkillers and fluid replacement and underwent renal artery embolization successfully 3 months post discharge from hospital. Seven months later the patient presented to her general practitioner with severe headache and was found to have a BP of $220 / 140 \mathrm{mmHg}$ and papilloedema. Over the following four days, the patient's BP was brought under control to $126 / 85 \mathrm{mmHg}$ with amlodipine $10 \mathrm{mg}$ once daily and she was subsequently discharged. Ultrasound of the kidneys confirmed the normal right kidney but revealed that the left kidney to be hypo echoic with a $3.4 \mathrm{~cm}$ remnant of the angiomyolipoma. These findings, combined with persistently raised plasma renin activity and plasma aldosterone levels suggested that a hypoxic left kidney is the cause of the patient's hypertension.

The patient then underwent a Dimercaptosuccinic acid (DMSA) scan of her kidneys, renal angiography and renal vein blood sampling. These revealed slight impairment of the renal function on the left side with no evidence of renal artery stenosis. Furthermore, they demonstrated a raised plasma renin activity on the left versus the right ( $2.33 \mathrm{vs} 0.87 \mathrm{ng} / \mathrm{ml} /$ hour) consistent with a hypoxic left kidney. Given the patients desire to start a family, she was commenced on Nifedipine LA 60mg once daily and this controlled her BP well at 118/80mmHg.

\section{Case 2}

A 43-year-old female postal worker was referred to our hypertension clinic because of severe resistant hypertension. Her BP was $167 / 11 \mathrm{mmHg}$ while on six different anti-hypertensive medications. She had a past medical history of multiple sclerosis causing dysarthria, bronchial asthma and depression. Clinical examination was not significant save for mild right loin tenderness. Renal magnetic resonance angiography revealed a right renal artery saccular aneurysm $(1.0 \times 0.6 \mathrm{~cm})$ and fibromuscular dysplasia renal artery stenosis. Given the patients history, the renal artery aneurysm was deemed to be at high risk of rupture so the patient was offered embolization with Ethylene Vinyl Alcohol Copolymer (Onyx) which is a thick polymer that hardens on contact with blood. During the procedure, a small quantity of Onyx was dislodged and embolized a distal segmental renal artery. The procedure was otherwise uneventful 
and the patient was discharged home with simple analgesia for loin pain. Four weeks after the procedure, the patient returned to clinic with a BP of $181 / 121 \mathrm{mmHg}$ with hypertensive retinopathy. The patient was admitted to hospital and her antihypertensive medications were uptitrated.

\section{Discussion}

To our knowledge, the two cases we have presented are the first to report malignant/severe hypertension secondary to targeted non-occlusive renal artery intervention. In the first case, the post-embolization onset of severe hypertension in a previously normotensive patient together with the finding of partial left renal ischaemia on CT and DMSA together with the raised left renal vein renin levels are strongly suggestive of a causal relationship between the intervention and subsequent hypertension. Similarly in the second case, the reported accidental embolization of a segmental artery and onset of malignant hypertension following the procedure are suggestive of a link. In hypothesising the link between RAE and hypertension, it is important to understand the potential role of the kidneys in development of essential hypertension.

Mild increase in BP is a common finding following RAE and may last up to 24 hours but is transient. ${ }^{5}$ Two reports of a similar phenomenon can be found in the literature, ${ }^{6,7}$ but with the important distinguishing factor that unlike the cases we presented, they underwent complete renal arterial occlusion for renal cell carcinoma. In hypothesising the link between RAE and hypertension, we will initially review the potential effects of reduced blood supply on the RAS and the ischemic induced microvascular changes that may underline hypertension. Although the kidney function is maintained over a wide range through auto-regulation, sustained reduction in renal perfusion can lead to disturbed microvascular function and vascular rarefaction. ${ }^{8}$

In the early 1970s, several studies on animals had shown that despite the activation of RAS by hypoperfusion, complete infarction of a kidney does not produce hypertension 1 presumably by eliminating renin secretion by the juxtaglomular apparatus. It was therefore thought that embolization could be used as an alternative to the more invasive surgical nephrectomy to reverse uncontrolled hypertension., ${ }^{2,9}$ Hom et al., ${ }^{10}$ postulated a similar premise when for the first time they raised hypertension as a potential complication of selective renal embolization. Emphasising the importance of complete renal artery embolization, they argued that otherwise ischaemia secondary to hypoperfusion of the distal vascular bed stimulates collaterals in turn up-regulating renin levels and potentiating hypertension. In the case of embolization induced hypertension reported by Alavi \& McLean ${ }^{6}$ post embolization angiogram showed collateral vessels supplying the tumour and substantially elevated renin levels on the side of the procedure on selective renal vein sampling. The resultant hypertension in these cases can therefore be attributed to incomplete infarction and distal ischaemia causing increased renal-angiotensin activity. In this regard we should consider changes at a microvascular level induced by ischaemia secondary to embolization.

Evidence for a primary role for microvascular abnormalities in the development of hypertension comes also from studies using antiangiogenic drugs e.g. vascular endothelial growth factor (VEGF) inhibitors in cancer patients. Hypertension is seen in up to $36 \%$ of patients on Bevacizumab and interestingly resolves with cessation of therapy. ${ }^{11}$ Mourad et al., ${ }^{12}$ found a significant reduction in capillary density following 6 months of treatment with Bevacizumab. There was a concomitant rise in the mean systolic and diastolic blood pressure in this time. Steeghs et al., ${ }^{13}$ reported similar findings following 5 weeks of treatment with another angiogenesis inhibitor, Telatinib.

The cases presented highlight the potential development of malignant hypertension following selective renal artery embolization. Diagnosis of malignant hypertension is of vital importance, given its poor prognosis if untreated. Monitoring of arterial blood pressure post renal embolization will enable recognition and early treatment of hypertension.

\section{Acknowledgements}

None.

\section{Conflicts of interest}

Author declares there are no conflicts of interest.

\section{Funding}

None.

\section{References}

1. Almgard LE, Ljungqvist A. Experimental occlusion of the renal circulation in the dog. Scand J Urol Nephrol. 1971;5:268-272.

2. Bergreen PW, Woodwide JR. Treatment of uncontrolled hypertension by therapeutic renal infarction. Urology. 1976;8(6):593-595.

3. Thompson JF, Fletcher EW, Wood RF, et al. Control of hypertension after renal transplantation by embolisation of host kidneys. Lancet. 1984;2(8400):424-427.

4. Russo D, Andreucci VE, Iaccarino V, et al. Percutaneous renal embolisation in renovascular hypertension. Br Med J (Clin Res Ed). 1988;296(6630):1160-1161.

5. Collins CS, Eggert CH, Stanson AJ, et al. Long-term follow-up of rena function and blood pressure after selective renal arterial embolization. Perspect Vasc Surg Endovasc Ther. 2010;22(4):254-260.

6. Alavi JB, McLean GK. Hypertension with renal carcinoma. An effect of arterial embolization. Cancer. 1983;52(1):169-172.

7. Probert CS, Osborn DE, Watkin EM. Malignant hypertension due to embolisation of a clear cell renal carcinoma. British Journal of Urology. 1992;70(1):95-96.

8. Textor SC, Lerman L. Renovascular hypertension and ischemic nephropathy. Am J Hypertens. 2010;23(11):1159-1169.

9. Millard FC, Hemingway AP, Cumberland DC, et al. Renal embolization for ablation of function in renal failure and hypertension. Postgrad Med J . 1989;65(768):729-734

10. Hom D, Eiley D, Lumerman JH, et al. Complete renal embolization as an alternative to nephrectomy. J Urol. 1999;161(1):24-27.

11. Kappers MH, van Esch JH, Sleijfer S, et al. Cardiovascular and renal toxicity during angiogenesis inhibition: clinical and mechanistic aspects. J Hypertens. 2009;27(12):2297-2309.

12. Mourad JJ, des Guetz G, Debbabi H, et al. Blood pressure rise following angiogenesis inhibition by bevacizumab. A crucial role for microcirculation. Ann Oncol. 2008; 19(5):927-934.

13. Steeghs N, Gelderblom H, Roodt JO, et al. Hypertension and rarefaction during treatment with telatinib, a small molecule angiogenesis inhibitor. Clin Cancer Res. 2008; 14(11):3470-3476. 